\title{
EFFECT OF DIFFERENT PRE-TREATMENTS ON THE PERMEABILITY OF GLUE-LAMINATED BAMBOO
}

\author{
Chunyan Lv, Caijuan Zhang, Xinje Zhou, Mingyu He, Lili Yu \\ Tianjin University Of Science And Technology \\ Faculty Of Light Industry Science And Engineering \\ P. R. CHINA \\ Q. R. China \\ Zhenzhong TANG \\ China Timber \& Wood Products Distribution Association \\ R. R. CHINA
}

(Received June 2020)

\begin{abstract}
In this study, hydrothermal treatments (duration: $2 \mathrm{~h}, 5 \mathrm{~h}, 8 \mathrm{~h}$; temperature: $60^{\circ} \mathrm{C}$, $80^{\circ} \mathrm{C}, 100^{\circ} \mathrm{C}$ ), ultrasonic treatments (duration: $60 \mathrm{~min}, 90 \mathrm{~min}, 120 \mathrm{~min}$; temperature: $40^{\circ} \mathrm{C}, 50^{\circ} \mathrm{C}, 60^{\circ} \mathrm{C}$; ultrasonic power: $400 \mathrm{~W}, 600 \mathrm{~W}, 800 \mathrm{~W}$ ) and freeze-drying treatments (vacuum degree: $0.05 \mathrm{mbar}, 0.1 \mathrm{mbar}, 0.5 \mathrm{mbar}, 1.0 \mathrm{mbar}, 1.7 \mathrm{mbar}$ ) were performed respectively to improve the permeability of glue-laminated bamboo. The effects of different pre-treatments on the permeability were compared according to the water absorption test and the mercury intrusion porosimetry test. The microstructure change of the samples was observed by scanning electron microscope (SEM). The results showed that freeze-drying treatment was an effective way to increase the permeability of the samples, in which the water absorption rate can be increased by $47 \%$, and the porosity can be increased by $10 \%$ at 0.5 mbar vacuum. From SEM analysis, some small holes appeared in the cell wall of the freeze-dried samples, because he free water inside the samples was changed into ice, and the volume became larger, and the pore diameter of the bamboo was enlarged.
\end{abstract}

KEYWORDS: Glue-laminated bamboo, permeability, hydrothermal treatment, freeze-drying, porosity.

\section{INTRODUCTION}

With the rapid development of China, the wooden resources cannot meet the increasing needs of the market, as the result, bamboo has been widely considered as the best substitute for wood because of the shorter growth cycle, the better mechanical properties and 
the rich accumulation (Ge et al. 2012, Seo et al. 2016, Kaur et al. 2016). However, the permeability of bamboo is very poor, which has obstructed the processing and modification treatments, such as dyeing, preservation, and flame-retardant treatment (Wen et al. 2017, Chen et al. 2019). As bamboo contains almost no lateral structure such as wood rays, the liquid flow can only pass through the pits (Ilic 1995, Sernek et al 1999), which is the main reason for the poor permeability of bamboo. When the bamboo-based material is subjected to permeation treatment, the pore diameter will increase and the quantity will increase. Therefore, improving the permeability of bamboo has become the key step for promoting the application of bamboo.

From the previous researches, some effective measures have been successfully performed to improve the permeability of bamboo including physical and chemical methods. The shortcomings of the chemical method are very obvious. For example, the mechanical properties of the material are greatly reduced, the color of the material is deepened, and the equipment is corroded. (Hass et al. 2012, Lu et al. 2017, Haase et al. 2018). The main physical methods include hydrothermal treatment, ultrasonic treatment, and freeze drying. The main purpose of hydrothermal treatment is to reduce the extract in bamboo (Xiao et al. 2017), thereby increasing the liquid circulation channel and improving the permeation. The principle is that some terpenoids in the extract which can be azeotroped in water at about $100^{\circ} \mathrm{C}$, resulting in the extracts leaching out from bamboo (Li et al. 2011, Kaur et al. 2013). Rao et al. (2013) showed that when bamboo was boiled at $80^{\circ} \mathrm{C}$ hot water for $2 \mathrm{~h}$, the penetration depth can be increased by $67 \%$ compared to the untreated samples. During ultrasonic treatment, water is used as the medium to generate shock waves inside the sample, so that the internal structure of the bamboo is destroyed and some weak tissues are also destroyed (Huang et al. 2017, Guan et al. 2019). Yong et al. (2013) have showed that the starch content in the bamboo with ultrasonic treatment at $70^{\circ} \mathrm{C}$ and $1400 \mathrm{~W}$ for $150 \mathrm{~min}$ has decreased significantly, and the average penetration depth was also increased from $431.33 \mu \mathrm{m}$ to $820.64 \mu \mathrm{m}$. Freeze-drying treatment has been proved as the most effective physical method at present, and the principle is involve in the three-phase morphological change of water. In the early phase, the water inside the sample began to freeze resulting in the pore size of bamboo enlarged in the second phase. In the third phase, the ice is directly sublimated and the pore diameter changed irreversibly ( $\mathrm{Lv}$ et al. 2005). The similar results also can be found in the research of $\mathrm{Xu}$ et al. (2018), which showed the porosity of bamboo with vacuum freeze-drying could increase to $73 \%$ and the mechanical properties have no noticeable change.

In this study, hydrothermal treatment, ultrasonic treatment, and freeze drying were also performed to improve the permeability of bamboo. The orthogonal test and single factor test were used to screen and compare the conditions of each method, and the optimal conditions of these three processes were obtained and compared. The mechanisms of permeability change were analyzed by the observations of the internal structure of the bamboo using a scanning electron microscope (SEM) and the pore size and pore size distribution of the specimen before and after treatment obtained from the mercury intrusion porosimeter (MIP).

\section{MATERIAL AND METHODS}

\section{Samples}

Glue-laminated bamboo hot pressed with bamboo strips was obtained from Ganzhou, Jiangxi Province and the moisture content was $8-10 \%$. The experimental specimens were cut from the gluelaminated bamboo with three dimensions of $20 \mathrm{~mm}$ (length) $\times 20 \mathrm{~mm}$ (width) $\times 7 \mathrm{~mm}$ (thickness) for water absorption test, $10 \mathrm{~mm}$ (length) $\times 10 \mathrm{~mm}$ (width) $\times 3 \mathrm{~mm}$ (thickness) for SEM analysis 
and $15 \mathrm{~mm}$ (length) $\times 15 \mathrm{~mm}$ (width ) $\times 7 \mathrm{~mm}$ (thickness) for MIP analysis. The glue-laminated bamboo with epoxy resin was manufactured by Jiangxi Anzhu Science and Technology Company and the cross section of the samples were sanded smooth enough although with the adhesive joints.

\section{Hydrothermal experiment}

The orthogonal experimental conditions of the glue-laminated bamboo with hydrothermal treatment were set in the following conditions: the treatment time was set in three levels of $2 \mathrm{~h}, 5 \mathrm{~h}$ and $8 \mathrm{~h}$, and the treatment temperature was set in three levels of $60^{\circ} \mathrm{C}, 80^{\circ} \mathrm{C}$ and $100^{\circ} \mathrm{C}$.

\section{Ultrasonic experiment}

The orthogonal experimental conditions of the glue-laminated bamboo with ultrasonic treatment were set in the following conditions: the treatment time was set in three levels of $60 \mathrm{~min}, 90 \mathrm{~min}$ and $120 \mathrm{~min}$, the temperature was set in three levels of $40^{\circ} \mathrm{C}, 50^{\circ} \mathrm{C}$ and $60^{\circ} \mathrm{C}$ and the ultrasonic power was set in three levels of $400 \mathrm{~W}, 600 \mathrm{~W}$ and $800 \mathrm{~W}$.

\section{Vacuum freeze-drying experiment}

Before vacuum freeze-drying treatment, the samples were immersed in water for several days to adjust the moisture content to 50-60\%. The vacuum freeze-drying method was performed using the vacuum freeze-drying machine (ALPHA 1-2 LD plus; Christ company of Germany) to dry the bamboo specimens and the condensation temperature was $-50^{\circ} \mathrm{C}$. In this experiment, the vacuum degree during vacuum drying was selected as the single variable, and was set in five levels of $1.7 \mathrm{mbar}(170 \mathrm{~Pa}), 1.0 \mathrm{mbar}(100 \mathrm{~Pa}), 0.5 \mathrm{mbar}(50 \mathrm{~Pa}), 0.1 \mathrm{mbar}(10 \mathrm{~Pa})$, $0.05 \mathrm{mbar}(5 \mathrm{~Pa})$. During the drying process, the drying time is $15 \mathrm{~h}$, and the drying temperature is $-15^{\circ} \mathrm{C},-20^{\circ} \mathrm{C},-27^{\circ} \mathrm{C},-42^{\circ} \mathrm{C},-48^{\circ} \mathrm{C}$ according to these five different vacuum degrees.

\section{Evaluation of permeability}

Before the water absorption test, the oven-dried method was performed in accordance with the national standard (GB/T 17657-2013) to determine the moisture content of gluelaminated bamboo. After different treatments, the samples were immersed in a beaker containing deionized water, and after $6 \mathrm{~h}$, each sample was weighed for the first time, and then weighed every $24 \mathrm{~h}, 48 \mathrm{~h}, 96 \mathrm{~h}$, and $128 \mathrm{~h}$, and the experimental results were recorded. The water absorption of the sample was calculated by using Eq. 1. This method was performed in accordance with the national standard (GB/T 1934.1 (2009):

$$
W A R=\frac{W_{1}-W}{W} \times 100 \%
$$

where: $\mathrm{W}$ and $\mathrm{W} 1$ were the weights of each sample before and after water absorption measurement, WAR is the water absorption ratio.

\section{Orthogonal experiment analysis}

This orthogonal experiment uses the L9 design table, and the factors for each condition are selected in three levels. According to the size of the $\mathrm{K}$ value in the range, the optimal conditions of the process are selected, and the influence of each factor on the experimental results is analyzed by the $\mathrm{R}$ value. 


\section{SEM analysis}

Scanning electron microscopy (JSM-IT300LV, Japan) were used to observe the microstructure of the experimental specimens. The samples $(10 \times 10 \times 3 \mathrm{~mm})$ with the best permeability performance in each treatment were selected and coated with gold under an environment of a vacuum of $5 \mathrm{~Pa}$ before the observation.

\section{Pore size analysis}

The mercury intrusion porosimetry experiment was performed using an automated mercury porosimeter (AutoPore IV 9510; USA) and the sample size is $15 \times 15 \times 7 \mathrm{~mm}$. The principle of MIP test is to use the characteristics of mercury to press the mercury into the interior of the bamboo under pressure conditions, so that the pore size and pore size distribution of the sample can be measured by the relationship between the total amount of mercury intrusion and the pore size.

\section{RESULTS AND DISCUSSION}

\section{Permeability analysis}

Hydrothermal treatment

The optimal conditions for the hydrothermal treatment were obtained by the analysis of the range and variance of the orthogonal experiments. As showed in Tab. 1, the Rang value $(\mathrm{R})$ of the temperature is much larger than the $\mathrm{R}$ value of the time, which indicated that during hydrothermal treatment, temperature has greater impact on the permeability of the treated gluelaminated bamboo. The reason may be attributed to the extracts in the samples much more easily removed out during hydrothermal treatment with higher temperature. For example, some terpene compounds can be azeotroped with water at $100^{\circ} \mathrm{C}$ and glycans and polyphenols are soluble in hot water (Li et al. 2011).

Tab. 1: The range results of hydrothermal treatment.

\begin{tabular}{cccc}
\hline Test No. & $\begin{array}{c}\text { Temperature } \\
\left({ }^{\circ} \mathbf{C}\right)\end{array}$ & $\begin{array}{c}\text { Time } \\
(\mathbf{h})\end{array}$ & $\begin{array}{c}\text { Water absorption ratio } \\
(\mathbf{\%})\end{array}$ \\
\hline 1 & 60 & 2 & 56.1 \\
2 & 60 & 5 & 56.8 \\
3 & 60 & 8 & 56.8 \\
4 & 80 & 2 & 60.0 \\
5 & 80 & 5 & 58.7 \\
6 & 80 & 8 & 57.9 \\
7 & 100 & 2 & 59.6 \\
8 & 100 & 5 & 61.8 \\
9 & 100 & 8 & 61.1 \\
K1 & 56.567 & 58.567 & \\
K2 & 58.867 & 59.1 & \\
K3 & 60.833 & 58.6 & \\
R & 4.266 & 0.533 & \\
\hline
\end{tabular}


According to the sum of the identified column with the correspondent factor $(\mathrm{K})$ based on the variance analysis as showed in Tab. 2, the optimum conditions were $100^{\circ} \mathrm{C}, 5 \mathrm{~h}$, which were the same as the results of the range analysis. The temperature had a significant effect at the 0.05 level, which also confirmed the importance of temperature during the hydrothermal treatment process. The water absorption ratio of the samples would significantly increase as the temperature increased. The results were similar with some previous results that Gao et al. (2015) processed the poplar by hydrothermal treatment. As the temperature increased, the reaction of poplar increased, and the permeability also increased.

Tab. 2: The variance results of hydrothermal treatment.

\begin{tabular}{lcccc}
\hline Factors & Sum of squares & DF & F-value & Sig. \\
\hline Temperature & 27.362 & 2 & 19.323 & $* *$ \\
Time & 0.536 & 2 & 0.379 & $/$ \\
Error & 1.42 & 2 & & \\
\hline
\end{tabular}

Note: / is referred to the factor insignificant at the 0.1 level, ${ }^{*},{ }^{* *},{ }^{* * *}$ are referred to the factor significant at the $0.1,0.05$, and 0.01 levels respectively.

\section{Ultrasonic treatment}

Based on the range analysis of ultrasonic experiments (Tab. 3), the order of influence factors on water absorption ratio during the ultrasonic treatment process was: time > ultrasonic power > temperature, which demonstrated that time was the most important factor on the permeability of bamboo laminated timber.

Tab. 3: The range results of ultrasonic experiment.

\begin{tabular}{ccccc}
\hline Test no. & $\begin{array}{c}\text { Temperature } \\
\left({ }^{\circ} \mathbf{C}\right)\end{array}$ & $\begin{array}{c}\text { Time } \\
(\mathbf{h})\end{array}$ & Ultrasonic power & $\begin{array}{c}\text { WAR } \\
\mathbf{( \% )}\end{array}$ \\
\hline 1 & 1 & 1 & 1 & 62.7 \\
2 & 1 & 2 & 2 & 61.8 \\
3 & 1 & 3 & 3 & 57.2 \\
4 & 2 & 1 & 2 & 59.6 \\
5 & 2 & 2 & 3 & 58.2 \\
6 & 2 & 3 & 1 & 57.4 \\
7 & 3 & 1 & 3 & 59.1 \\
8 & 3 & 2 & 1 & 65.8 \\
9 & 3 & 3 & 2 & 59.1 \\
K1 & 60.567 & 60.467 & 61.967 & \\
K2 & 58.4 & 61.933 & 60.167 & \\
K3 & 61.333 & 57.9 & 60.867 & \\
R & 2.933 & 4.033 & 3.8 & \\
\hline
\end{tabular}

Guan et al. (2012) used ultrasonic treatment of bamboo, and the results showed that the higher the power, the longer the time, the more substances were extracted from the bamboo. The difference in this experiment is that the maximum power of the ultrasonic instrument used 
is much smaller than the power level selected for the above experiment, and the main effect is not the power level but the time. Based on the variance analysis of ultrasonic experiments (Tab. 4), the optimal conditions were $60^{\circ} \mathrm{C}, 90 \mathrm{~min}$, and $400 \mathrm{~W}$. However, the three factors selected in the experiment were not significant, and the influence order of each factor was the same as that in the range analysis.

Tab. 4: The range results of ultrasonic experiment.

\begin{tabular}{lcccc}
\hline Factors & Sum of squares & Degree of Freedom & F-value & Sig. \\
\hline Temperature & 13.887 & 2 & 4.441 & $/$ \\
Time & 25.007 & 2 & 7.997 & $/$ \\
Ultrasonic power & 21.680 & 2 & 6.933 & $/$ \\
Error & 3.13 & 2 & & \\
\hline
\end{tabular}

Vacuum freeze-drying experiment

Percentages of water absorption in the glue-laminated bamboo increased as the duration prolonged during vacuum freeze-drying treatment under $0.05,0.1,0.5,1.0$, and 1.7 mbar, respectively as showed in Fig. 1a.

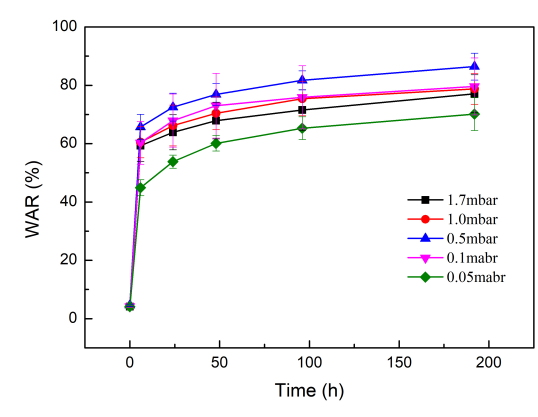

(a)

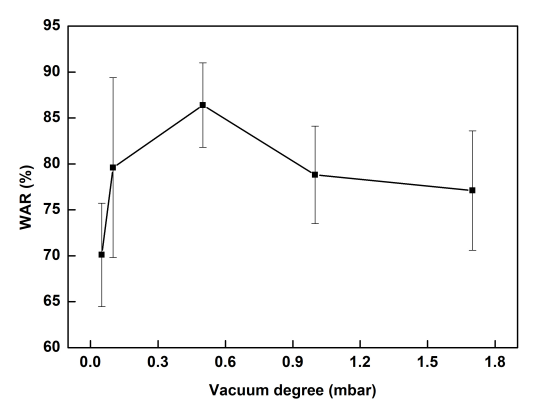

(b)

Fig. 1: Percentage of water absorption of the treated bamboo after different vacuum freeze-drying experiments: (a )the treatment process, (b) the maximum water absorption ratio.

The water absorption ratios under these five vacuum degrees were higher in the first $10 \mathrm{~h}$, and then the growth rate became slow. In the first $6 \mathrm{~h}$, the water absorption rate of each group was larger, and the water absorption rate increased rapidly. Then, with the passage of time, the water absorption rate gradually decreased. At $192 \mathrm{~h}$, the water absorption rate of the 0.5 mbar group was the highest, and the water absorption rate was $5 \%-16 \%$ higher than that of the other groups. In the subsequent experiments, 0.5 mbar was selected as the vacuum degree when the bamboo was dried. The reason for this phenomenon may be that too low a vacuum will make the sublimation process difficult to carry out, and too high a vacuum, the drying temperature is higher, so that the pore diameter of the bamboo material rebounds.

The comparison of the maximum water absorption ratio in the glue-laminated bamboo under these five vacuum degrees were shown in Fig. 1b. It can be seen that the water absorption ratio in the glue-laminated bamboo was increased as the vacuum degree increased from 0.05-0.5 mbar during vacuum freeze-drying treatment, and began to decrease when the vacuum degree was higher than 0.5 . When the vacuum degree between $0.05 \mathrm{mbar}$ and $0.5 \mathrm{mbar}$, 
as the vacuum degree increased, the vapor pressure on the surface of the dried layer of treated bamboo was decreased, so that the crystallization inside the bamboo can be directly sublimated in a large volume, and the permeation effect is increased. From 0.5-1.7 mbar, when the vacuum degree increased, the surface of sublimation temperature would be lowered significantly, which has resulted in the decrease of the internal crystallization of the treated bamboo, and the originally enlarged pore size was rebounded, as the result the permeability became poor.

\section{Comparison of different treatments on the water permeability}

The optimal conditions of these three methods, hydrothermal experiment (HE), ultrasonic experiment (UE), vacuum freeze-drying experiment (VE) and the untreated sample (US) were showed in Fig. 2.

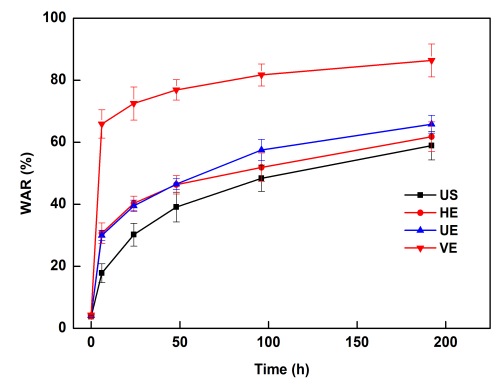

Fig. 2: Water absorption rate under four conditions: hydrothermal experiment (HE), ultrasonic experiment (UE), vacuum freeze-drying experiment (VE) and the untreated sample (US).

It was noted that the water absorption rate of the treated wood after different treatments was improved significantly compared to the control group. Among of these three treatments, the freeze-drying group had the best performance for improving the permeability of treated wood, and the water absorption rate could be increased by $47 \%$ compared to the control group. While hydrothermal and ultrasound treatments were not very promising, only increased by $5 \%$ and $12 \%$ resp. From the growth trend of the water absorption rate during $200 \mathrm{~h}$, in the first $10 \mathrm{~h}$, the water absorption rate of each group was increased sharply, then it would become gradually reduced, and after $150 \mathrm{~h}$, the water content in the samples gradually became saturated.

In the hydrothermal treatment, due to the decrease in the extract, the obstruction substance in the passage inside the bamboo is reduced, and the permeation effect is improved. However, the disadvantage of this method is that, in addition to the extract which is soluble in boiling water, other types of extracts cannot be removed, resulting in an infiltration effect (Bao and Zhou 2017). Ultrasonic method relies on external force to cause cell wall damage and increase the penetration channel of bamboo. According to the observation results of SEM, the instrument used has less damage to the cell wall, which leads to poor penetration. The freeze-drying method uses the principle that the same mass of water becomes ice, and the volume becomes larger, and the pore diameter is enlarged inside the permeation channel, thereby increasing the permeation channel. From the experiment, compared with the former two methods, the effect is very obviously (Han 2007). 


\section{Mercury intrusion porosimeter analysis}

According to Tab. 5, the total intrusion volume of the control sample was only $0.48 \mathrm{~mL} \cdot \mathrm{g}^{-1}$, while it would be increased by about $83 \%$ of the sample with freeze drying treatment. The reason was that a series of holes appeared on the freeze-dried sample as observed by SEM (Fig. 3), which became the channels to the bamboo inside, as the result more mercury could be pressed into the glue-laminated bamboo.

Tab. 5: MIP analysis of glue-laminated bamboo with different treatment conditions.

\begin{tabular}{lcccc}
\hline Sample & $\begin{array}{c}\text { Total intrusion } \\
\text { volume } \\
\left(\mathbf{m L} \cdot \mathbf{g}^{-\mathbf{1}}\right)\end{array}$ & $\begin{array}{c}\text { Porosity } \\
\mathbf{( \% )}\end{array}$ & $\begin{array}{c}\text { Median pore } \\
\text { diameter } \\
\mathbf{( n m})\end{array}$ & $\begin{array}{c}\text { Bulk density } \\
\left(\mathbf{g} \cdot \mathbf{c m}^{-3)}\right.\end{array}$ \\
\hline Control & 0.48 & 41.20 & 26.30 & 0.87 \\
Freeze drying & 0.87 & 51.80 & 50.40 & 0.60 \\
Ultrasound & 0.57 & 50.10 & 38.60 & 0.79 \\
Hydrothermal & 0.53 & 36.60 & 45.40 & 0.69 \\
\hline
\end{tabular}

From the porosity results, compared with the control group, the median pore size of the three methods has increased. It can be seen that the porosity of untreated samples was quite low, which was the main reason for the poor permeability of bamboo, and the biggest porosity was only increased to $51.8 \%$ observed in the samples with the promising freeze drying treatment. The mercury intrusion volume of the ultrasound and the hydrothermal samples also increased with respect to the control samples, but the porosity of the hydrothermal group was lower than that of the control samples. The reason may be attributed that the porosity was derived from the volume of mercury injected/sample volume (Plötze et al. 2011, Yang et al. 2013). When the sample was selected, the sample may be selected from the bamboo plate at a different position. The selected sample has a larger volume, which results in a smaller porosity. Compared with the control group, hydrothermal methods and ultrasonic methods increased the volume of mercury by $11 \%$ and $20 \%$.

The pore diameters of the freeze-dried samples were mainly about $40-50 \mathrm{~nm}$ as observed in Tab. 5, and the median diameter of the group was $50.40 \mathrm{~nm}$. The ultrasound group had the largest difference from the control group at around $50 \mathrm{~nm}$ and $7 \mathrm{~nm}$, and its general trend was similar to that of the freeze-dried group. The difference between the hydrothermal group and the control group was the most obvious at $25 \mathrm{~nm}$, which indicated that the hydrothermal treatment increased the number of pores around $25 \mathrm{~nm}$, but the amount was still less than that of the freeze-dried group. It was further verified that the permeation effect was not as good as that of the freeze-drying group. It shows that in the range of $40-50 \mathrm{~nm}$, the distribution of the pore size is the most extensive. At a pore size after $76 \mathrm{~nm}$, the volume difference of the pressed mercury is small, which is about $75 \%$ lower than the range of the previous mesopores $(2-50 \mathrm{~nm})$, and then the curve tends to be moderated. The relationship between the cumulative mercury intrusion volume and pore size of glue-laminated bamboo with different treatments were shown in Fig. 3.

In general, the samples with different modification treatments could improve the total intrusion volume significantly compared to control samples. As observed in Fig. 3, the pore diameters of the samples with different treatments for mercury intrusion were mainly $10-100 \mathrm{~nm}$, and the cumulative mercury intrusion volume would decrease to a low level and change gently until the end when the pore size exceeded $100 \mathrm{~nm}$. 


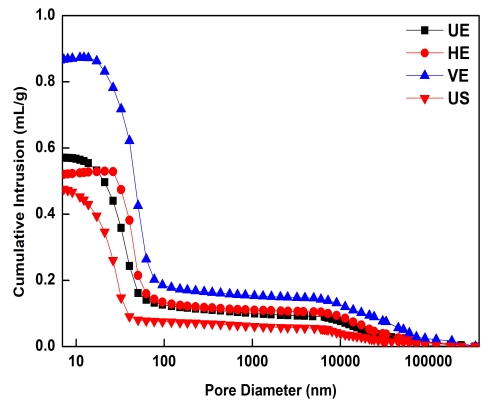

Fig. 3: Relationship between cumulative intrusion volume and pore diameter of glue-laminated bamboo with different treatment conditions.

It seemed that the cumulative mercury intrusion volume was bigger in the samples with different modification treatments compared to the control samples during mercury intrusion, and the promising result was observed in the freeze-dried samples, in which the cumulative intrusion volume was about $0.19-0.85 \mathrm{~mL} \cdot \mathrm{g}^{-1}$ in the range of $10-100 \mathrm{~nm}$, while it was only about $0.09-0.45 \mathrm{~mL} \cdot \mathrm{g}^{-1}$.

\section{Analysis of microstructure}

Microstructures of the samples before and after different treatments were evaluated by SEM as showed in Figs. 4a-d. The structure of bamboo was mainly composed of some thin-walled tissues and tube bundle structures. It can be seen that the parenchyma cells of the untreated samples were relatively intact, and the structures were relatively dense as observed in Fig. 4a. While the other samples with different modification treatments behaved different structural changes.

For the freeze-dried samples as showed in Fig. 4b, some small holes appeared on the cell wall, which was due to that after freezing treatment the internal free water of the samples could be turned into ice, and the volume became larger, as the result the pore diameter of the glue-laminated bamboo could be enlarged, and then it was dried, and directly sublimated to ice, ensuring that the enlarged aperture does not rebound and return to its original state (Ilic et al. 1995).

For the ultrasonically treated samples as showed in Fig. 4c, it can be clearly seen that the cracks appeared on the cell wall of glue-laminated bamboo, and some holes with different sizes appeared on the parenchyma cells, which indicated that using water as a medium, ultrasonic waves can break the parenchyma tissue of glue-laminated bamboo. As the result, the perforated film provided channels for water intrusion.

For the hydrothermally treated samples as showed in Fig. 4d, it can be seen that the whole cell does not change at all compared to the control samples. The hydrothermal treatment was generally in the higher temperature condition, and used water as the medium to enlarge the pore size of the glue-laminated bamboo (Bao and Zhou 2017). During the treatment, the extracts were boiled out or evaporated. However, the effect of hydrothermal treatment on the enlarging pore size or increasing the number of pores was less than the other two modification treatments. 


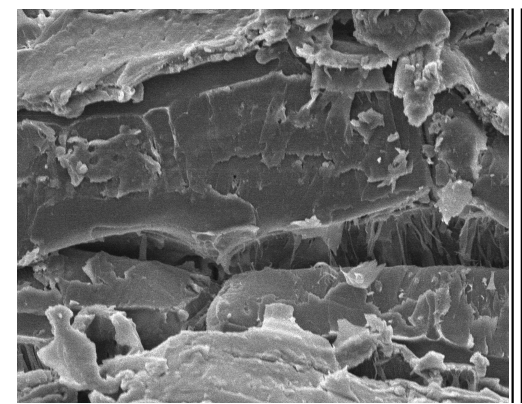

(a)

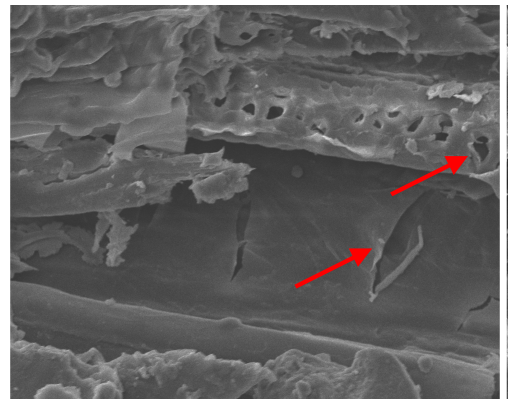

(c)

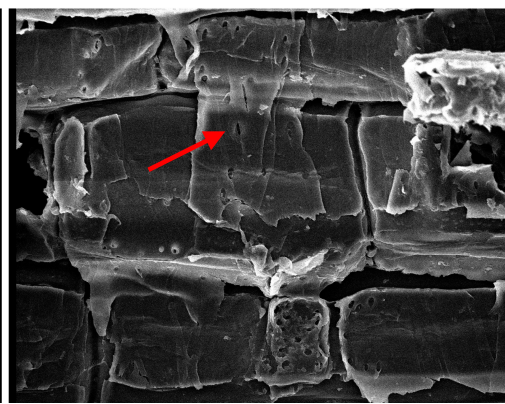

(b)

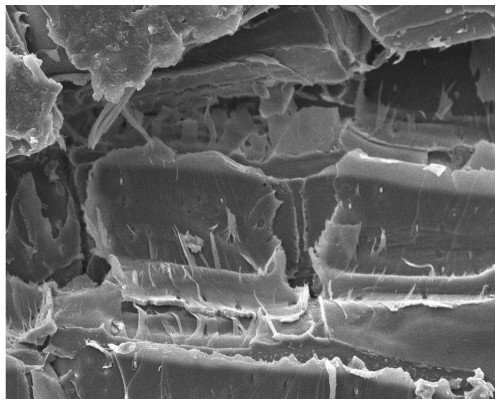

(d)

Fig. 4: Microstructure of bamboo before and after treatment: a) HS-1000×, b) VE-1000x, c) UE-1000×, d) $H E-1000 \times$.

\section{CONCLUSIONS}

The treatment effect of hydrothermal method is limited to the fact that many extracts cannot be extracted in the boiling water. The ultrasonic treatment effect is limited to the lower ultrasonic power of the selected instrument. The freeze-drying method has a good effect on the treatment of bamboo laminated materials. When the degree is $0.5 \mathrm{mbar}$, the water absorption rate can be optimized.

The mercury intrusion porosimetry relates the pore size distribution to the water absorption rate. The media pore size of the hydrothermal method $(45.40 \mathrm{~nm})$, the ultrasonic method $(38.60 \mathrm{~nm})$, and the freeze-drying method $(50.40 \mathrm{~nm})$, compared with the control group $(26.3 \mathrm{~nm})$, have increased by $73 \%, 47 \%, 92 \%$. Under the scanning electron microscope, both the ultrasonic method and the freeze-drying method can see the permeation channel after the external force is destroyed, and a series of micropores appear.

\section{ACKNOWLEDGMENT}

The authors are grateful for the financial support of the Subjects of National Natural Science Foundation of China (NSFC No. 31400499), the National College Students Innovation and 
entrepreneurship training program (201910057047), and the Science and Technology of Tianjin University College Students Innovation and entrepreneurship training program (202110057013).

\section{REFERENCES}

1. Bao, Y., Zhou, Y., 2017: Comparison between superheated steam drying and conventional drying of Chinese cedar lumber. Scientia Silvae Sinicae 53(1): 88-93.

2. Chen, H., Zhang, Y.T., Xue, Y., Han, J., Zhong, T.H., Wang, G., 2019: A comparative study of the microstructure and water permeability between flattened bamboo and bamboo culm. Journal of Wood Science 65(1): 1-14.

3. Emaminasab, M., Tarmian, A., Pourtahmasi, K., Avramidis, S., 2016: Improving the permeability of Douglas-fir (Pseudotsuga menziesii) containing compression wood by Physisporinus vitreus and Xylaria longipes. International Wood Products Journal 7(3): 110115.

4. Ge, H., 2012: A research on the apply of bamboo elements in packing design. Pp 24-32, China Academy of Art, Hangzhou, China.

5. Gao C., 2015: Study on the process and properties of hydrothermally treated poplar wood raw materials and its plate making. Master's thesis. Pp 25-34, Nanjing Forestry University, Nanjing, China.

6. Guan, M.J., Zhang, Z.W., Yong, C., Du, K.K., 2019: Interface compatibility and mechanisms of improved mechanical performance of starch/poly (lactic acid) blend reinforced by bamboo shoot shell fibers. Journal of Applied Polymer Science 136(35): 47899.

7. Hass, P., Wittel, F.K., Mendoza, M., 2012: Adhesive penetration in beech wood: experiments. Wood Science and Technology 46(1-3): 243-256.

8. Haase, J., Leung, L., Evans, P., 2018: Plasma pre-treatments to improve the weather resistance of polyurethane coatings on Black spruce wood. Coatings 9(1): 8-22.

9. Han, N., 2007: Progress of study on vacuum freeze-drying technology. Food Engineering (03): 28-29+47.

10. Huang, Z., 2017: Effect of ultrasonic treatment on surface properties and bonding properties of bamboo. Pp 49-55, Nanjing Forestry University, Nanjing, China.

11. Ilic, J., 1995: Advantages of prefreezing for reducing shrinkage-related degrade in eucalyptus: General considerations and review of the literature. Wood Science \& Technology 29(4): 277-285.

12. Kaur, P.J., Pant, K.K., Naik, S.N., 2016: Field investigations of selectively treated bamboo species. European Journal of Wood and Wood Products 74(5): 771-773.

13. Kaur, P., Kardam, V., Pant, K.K., 2013: Scientific investigation of traditional water leaching method for bamboo preservation. Bamboo science and culture. Journal of the American chemical society, 23(1): 27-32.

14. Li, Y., Liu, Y., Wang, F., 2011: Control factors and improvement measures of wood permeability. Scientia Science and Technology 47(5): 131-139.

15. Lu, C., Jiang, T., Liu, Y., 2017: Research progress on the influence factors of eucalyptus wood permeability and its improvement methods. Journal of Southwest Forestry University (Natural Science) 37(1): 214-220.

16. Lv, J.X., Li, Z.Y., Jiang, J.L., 2005: Liquid penetration of freeze-drying and air-drying wood of plantation Chinese fir. Forestry Study 16(4): 293-295.

17. Plötze, M., Niemz, P., 2011: Porosity and pore size distribution of different wood types as determined by mercury intrusion porosimetry. European Journal of Wood and Wood Products 69(4): 649-657. 
18. Rao, S., Yu, L., 2013: Effect of pretreatment on fluid permeability of bamboo. Forestry Machinery \& Woodworking Equipment 41(8): 26-29.

19. Sernek, M., Resnik, J., Kamke, F.A., 1999: Penetration of liquid urea-formaldehyde adhesive into beech wood. Wood and Fiber Science 31(1): 41-48.

20. Seo, H.J., Kim, S., Huh, W., 2016: Enhancing the flame-retardant performance of wood-based materials using carbon-based materials. Journal of Thermal Analysis and Calorimetry 123(3): 1935-1942.

21. Wen, X., 2017: Study on the penetration and influence factors of preservatives in bamboo. Pp 43-49, Chinese Academy of Forestry, Beijing, China.

22. Xu, J., He, S., Li, J., 2018: Effect of vacuum freeze-drying on enhancing liquid permeability of Moso bamboo. BioResources 13(2): 4159-4174.

23. Xiao, H., 2017: Microwave treatment of Pinus sylvestris var. Mongolica. Pp 89-92, Chinese Academy of Forestry, Beijing, China.

24. Yang, F., Ning, Z., Kong, D., Liu, H., 2013: Analysis of shale pore structure by high pressure mercury intrusion and nitrogen adsorption. Natural Gas Geosciences 3: 450-455.

25. Yong, C., Chan, X., Guan, M., 2013: Effect of ultrasonic wall breaking effect on transverse permeability of bamboo. Bamboo Research Summary 32(4): 33-36+41.

Chunyan Lv, Caijuan Zhang, Xinjie Zhou, Mingyu He, Lili Yu*

Tianjin University Of Science And Technology

P. R. CHINA

Z HenZHONG TANG*

China Timber \& Wood Products Distribution Association

P. R. CHINA

Chunyan Lv, Caijuan Zhang, Xinjie Zhou, Mingyu He, Lili Yu* Tianjin University Of Science And Technology

Faculty Of Light Industry Science And Engineering

Dagu Southroad 1038, Hexi District, P. O. Box 546

300222 Tianjin

Q. R. China

Z HenZHONG TANG*

China Timber \& Wood Products Distribution Association

R. R. CHINA

Lugu Road 74, Shijingshan District

100040 BEIJING

*Corresponding author: yulilucky@tust.edu.cn

*Corresponding author: zhenzhongtang@hotmail.com 